Bull. Austral. Math. Soc.

$54 \mathrm{D} 30$

VOL. 38 (1988) [393-395]

\title{
A NOTE ON COMPACT SETS IN SPACES OF SUBSETS
}

\author{
Phil Diamond and Peter Kloeden
}

\begin{abstract}
A simple characterisation is given of compact sets of the space $\mathcal{K}(X)$, of nonempty compact subsets of a complete metric space $X$, with the Hausdorff metric $d_{H}$. It is used to give a new proof of the Blaschke selection theorem for compact starshaped sets.
\end{abstract}

Let $\left(\mathcal{E}^{n}, D\right)$ be the metric space of fuzzy sets on $\mathbf{R}^{n}$, with $D$ the supremum over Hausdorff distances between corresponding level sets. Compact sets of this space have recently been characterised [2]: roughly speaking, a closed set $\mathcal{U}$ of $\left(\mathcal{E}^{n}, D\right)$ is compact if and only if

(1) fuzzy sets in $\mathcal{U}$ have uniformly bounded support, and

(2) the level set maps $\alpha \mapsto[u]^{\alpha}=\left\{x \in \mathbf{R}^{n}: \alpha \leqslant u(x) \leqslant 1\right\}, 0<\alpha \leqslant 1$, are uniformly equi-leftcontinuous for all $u \in \mathcal{U}$.

The Blaschke selection theorem and its converse follow as a simple corollary: that compact sets of the metric space $\left(\mathcal{K}_{c o}^{n}, d_{H}\right)$ of nonempty compact convex subsets of $\mathbf{R}^{n}$, where $d_{H}$ is the induced Hausdorff metric, are the closed, uniformly bounded subsets of $\mathcal{K}_{c o}^{n}$. Such subsets are represented as fuzzy sets by their characteristic functions. These are independent of $\alpha$, so condition (2) is automatically satisfied.

On the other hand, it is well-known that if $(X, d)$ is a compact space, then so too is $\left(\mathcal{K}(X), d_{H}\right)$, the space of nonempty compact subsets of $X$ with metric $d_{H}$ [3]. Thus $B$ compact in $X$ gives $\mathcal{K}(B)$ compact in $\mathcal{K}(X)$. However, converse theorems (which then characterise compact subsets of $\mathcal{K}(X))$ have apparently not been stated. Such results are not without application, because frequently a subset of $\mathcal{K}(X)$ is studied, rather than the whole space, as in formulating concepts of random sets $[4,5]$. We present below a direct proof of such a characterisation, which is more convenient than the usual one involving total boundedness. As a direct application, Corollary 2 is a new proof of the Blaschke theorem and its converse for starshaped sets, without gauge functions [1].

Definition: A nonempty subset $\mathcal{U}$ of $\left(\mathcal{K}(X), d_{H}\right)$ is said to be compactly bounded (in $X$ ) if there exists a compact subset $W$ of $X$ such that $U \subseteq W$ for every $U \in \mathcal{U}$.

Received 8 February 1988

Copyright Clearance Centre, Inc. Serial-fee code: 0004-9729/88 \$A2.00+0.00. 
Proposition. Let $(X, d)$ be a complete metric space. Then a nonempty closed subset $\mathcal{C}$ of $\left(\mathcal{K}(X), d_{H}\right)$, the metric space of nonempty compact subsets of $X$, is compact if and only if $\mathcal{C}$ is compactly bounded.

Corollary 1. A nonempty closed subset $\mathcal{C}$ of $\left(\mathcal{K}\left(\mathbf{R}^{n}\right), d_{H}\right)$ is compact if and only if $\mathcal{C}$ is uniformly bounded in $\mathbf{R}^{n}$.

COROLlaRY 2. Let $\mathcal{V}$ be a nonempty closed subset of the class of nonempty compact starshaped sets in $\mathbf{R}^{n}$. Then $\mathcal{V}$ is compact if and only if $\mathcal{V}$ is uniformly bounded in $\mathbf{R}^{n}$.

Proof of Proposition: Write $W(\mathcal{C})=\bigcup\{C: C \in \mathcal{C}\}$. If $W(\mathcal{C}) \subseteq V$, where $V$ is compact in $X$, then $\mathcal{C} \subseteq \mathcal{K}(V) \subseteq \mathcal{K}(X)$, and $\mathcal{C}$ is closed in the subspace topology on $\mathcal{K}(V)$. Thus $\mathcal{C}$ is compact in $\mathcal{K}(V)$, and in $\mathcal{K}(X)$. Conversely, if $\mathcal{C}$ is compact in $\left(\mathcal{K}(X), d_{H}\right)$ then it is also sequentially compact. Let $\left\{x_{i}\right\}$ be a sequence in $W(\mathcal{C})$. Then there is a corresponding sequence of sets $\left\{C_{i}\right\}$ in $\mathcal{C}$, such that $x_{i} \in C_{i}$ for each $i$. Compactness of $\mathcal{C}$ gives a subsequence $\left\{C_{i(j)}\right\}$ converging to $C_{0} \in \mathcal{C}$. So $d\left(x_{i(j)}, C_{0}\right) \rightarrow 0$ as $i(j) \rightarrow \infty$. Since $C_{0}$ is itself compact, there is a further subsequence $\left\{x_{k^{\prime}}\right\} \subseteq\left\{x_{i(j)}\right\}$ converging to some $x_{0} \in C_{0}$. Thus $W(\mathcal{C})$ is a sequentially compact subset of $X$, and $\mathcal{C}$ is compactly bounded.

Proof of Corollary 2: Let $\left\{C_{k}\right\}$ be a bounded sequence of compact starshaped sets in $\mathcal{V}$. A subsequence $\left\{C_{k^{\prime}}\right\} \subseteq\left\{C_{k}\right\}$ converges if and only if, for any bounded sequence of points $p_{k} \in \mathbf{R}^{n}$, a subsequence of $\left\{C_{k}-p_{k}\right\}$ converges. Thus it suffices to consider only sequences where each $C_{k}$ is starshaped with respect to the origin $O$. From Corollary 1 it only remains to show that the (relabelled) subsequence $\left\{C_{k}\right\}$ has limit $C \in \mathcal{K}\left(\mathbf{R}^{n}\right)$ which is starshaped. If $C$ were not starshaped, there would exist $y \in C, z \notin C$ such that $z \in$ the line segment $O y$. Recall that the Hausdorff semidistance $\rho(A, B)=\sup _{a \in A} \inf _{b \in B}\|a-b\|$ has the properties

$$
\begin{gathered}
\rho(A, B)=0 \text { if and only if } A \subseteq B \\
\rho(A, C) \leqslant \rho(A, C)+\rho(B, C)
\end{gathered}
$$

for sets in $\mathcal{K}\left(\mathbf{R}^{n}\right)$. By compactness, there exists $\eta>0$ such that $\rho(z, C)=\eta$. For $k$ sufficiently large, $\rho\left(C_{k}, C\right) \leqslant d_{H}\left(C_{k}, C\right)<\eta / 3$, so select $y_{k} \in C_{k}$ such that $\left\|y-y_{k}\right\|<\eta / 3$. Then there exists $z_{k} \in O y_{k} \subset C_{k}$ such that $\left\|z-z_{k}\right\|<\eta / 3$. Thus

$$
\eta=\rho(z, C) \leqslant \rho\left(z, z_{k}\right)+\rho\left(z_{k}, C_{k}\right)+\rho\left(C_{k}, C\right)<\eta / 3+0+\eta / 3,
$$

a contradiction, and $C$ is starshaped. 
Note. This characterisation of compact sets is not true for $\left(2^{X}, d_{H}\right)$, where $2^{X}$ is the set of nonempty closed subsets of $X$, when $X$ is not compact. For example, $\mathcal{C}=\left\{C_{\gamma}=\right.$ $[\gamma, \infty): 0 \leqslant \gamma \leqslant 1\}$ is a compact subset of $\left(2^{\mathbf{R}}, d_{H}\right)$, as $d_{H}\left(C_{\gamma}, C_{\beta}\right)=|\gamma-\beta|$ and thus $\mathcal{C}$ is totally bounded in $2^{\mathbf{R}}$. However, $\underset{0 \leqslant \gamma \leqslant 1}{\bigcup} C_{\gamma}=\mathbf{R}^{+}$is not a compact subset in $\mathbf{R}$ and $\mathcal{C}$ is not compactly bounded.

\section{REFERENCES}

[1] G. A. Beex, 'Starshaped sets and the Hausdorff metric', Pacific J. Math. 61 (1975), 21-27.

[2] P. Diamond and P. Kloeden, 'Characterization of compact subsets of fuzzy sets', Fuzzy Sets and Systems (1989) (to appear).

[3] F. Hausdorff, Set Theory (Chelsea Press, New York, 1957).

[4] G. Matheron, Random Sets and Integral Geometry (John Wiley, New York, 1975).

[5] W. Weil, 'An application of the central limit theorem for Banach-space-valued random variables to the theory of random sets', $Z$. Wahrsch. Verw. Gebiete 60 (1982), 203-208.

Dr P. Diamond

Mathematics Department

University of Queensland

St. Lucia, Qld 4067

Australia.
Dr P. Kloeden

School of Mathematical and Physical Sciences Murdoch University

Murdoch, W.A. 6150

Australia. 